\title{
Experiments on Intercontinental Haptic Control of Multiple UAVs
}

\author{
Martin Riedel ${ }^{1}$, Antonio Franchi ${ }^{1}$, Paolo Robuffo Giordano ${ }^{1}$, \\ Heinrich H. Bülthoff ${ }^{1,2}$, and Hyoung Il Son ${ }^{1}$ \\ 1 Max Planck Institute for Biological Cybernetics, \\ Spemannstrasse 38, 72076 Tübingen, Germany \\ 2 Department of Brain and Cognitive Engineering, \\ Korea University, Seoul, 136-713 Korea \\ \{martin.riedel, antonio.franchi,prg, hhb, hyoungil.son\}@tuebingen.mpg.de
}

\begin{abstract}
In this paper we propose and experimentally validate a bilateral teleoperation framework where a group of UAVs are controlled over an unreliable network with typical intercontinental time delays and packet losses. This setting is meant to represent a realistic and challenging situation for the stability the bilateral closed-loop system. In order to increase human telepresence, the system provides the operator with both a video stream coming from the onboard cameras mounted on the UAVs, and with a suitable haptic cue, generated by a force-feedback device, informative of the UAV tracking performance and presence of impediments on the remote site.

In addition to the theoretical background, we describe the hardware and software implementation of this intercontinental teleoperation: this is composed of a semi-autonomous group of multiple quadrotor UAVs, a 3-DOF haptic interface, and a network connection based on a VPN tunnel between Germany and South Korea. The whole software framework is based upon the Robotic Operating System (ROS) communication standard.
\end{abstract}

\section{Introduction}

Unmanned Aerial Vehicles (UAVs) represent a promising robotic platform for a large spectrum of current and future applications such as, e.g., indoor and outdoor surveillance, exploration of hazardous areas, search and rescue, and transportation [1. In recent years, considerable research efforts have been spent towards the use of multiple UAVs for addressing some of the aforementioned scenarios. In fact, use of multiple flying robots results in increased flexibility and robustness to single robot failures 2. However, designing decentralized algorithms for the control of multiple robots with high degree of autonomy is still a challenging task both from the theoretical and actual implementation side, although there have been big achievements from this perspective 34 .

Especially, a major difficulty is the realization of full autonomy in unstructured/unpredictable environments. The use of bilateral teleoperation (see, e.g., 5] 
for a review) represents in this sense an interesting possibility for improving the performance of a multi-UAV system with the contribution of the human operator's intelligence, i.e., exploiting the human-in-the-loop control. Recently, in this context, there have been several works aimed at developing different bilateral teleoperation algorithms to control multiple robots in an efficient and robust way 678 .

In an intercontinental bilateral teleoperation scenario, very large time delays (usually, in the range of $100 \mathrm{~ms}$ ) between the operator and slave sides represent one of the major difficulties for both theoretical (i.e., stability) and practical issues (e.g., quality of visual feedback, safety and easiness of maneuvering). The first intercontinental tele-surgical operation (without force feedback) was established between New York, USA, and Strasbourg, France, in 2001 [9. Recently [10] proposed a protocol to bilaterally teleoperate various haptic devices and slave robots located in different countries.

In long-distance teleoperation, the transmission of visual data is usually more delayed than the transmission of position/force data, because of the large size of video packets. This problem has been investigated in 11] by simulating a visual feedback in the operator site through an intercountry experiment. To the best of our knowledge, however, there was no result in the bilateral teleoperation of multi-robot UAV systems which were truly communicating over an intercontinental Internet connection.

In this paper, we aim at illustrating a software platform and experimental testbed that provides a standardized interface for the bilateral teleoperation of multiple robots (UAVs in our case). The UAVs autonomously keep a desired formation and avoid obstacles while being stably tele-operated by a the remote user. The theoretical framework used in this work, related to the one presented in [7, is then verified through a real intercontinental experiment between Germany and South Korea, thereby stressing in real conditions the robustness of our theoretical claims and software design.

The paper is organized as follows. The haptic teleoperation control framework is reviewed in Sect. 2. In Sect. 3 the proposed software platform is presented with a detailed description of hardware setup for the experiment. Following this, experimental results are reported and discussed in Sect.4. Finally, the concluding remarks and directions for future research are presented in Sect. 5.

\section{Haptic Interaction with Multiple UAVs}

In the presented framework the human operator is provided with three kind of interfaces: i) a console used to supervise the group of UAVs (e.g., to switch between different UAV control algorithms/behaviors), ii) a screen providing the video streams of the remote environment from different perspectives (e.g., onboard vs. fixed to the wall), and iii) a haptic device in order to intervene on the overall motion of the group and to receive a force feedback informative of the presence of obstacles and other environmental (e.g., aerodynamical) disturbances. 
The haptic device is a generic 3-DOF mechanical system:

$$
M(\boldsymbol{x}) \ddot{\boldsymbol{x}}+C(\boldsymbol{x}, \dot{\boldsymbol{x}}) \dot{\boldsymbol{x}}=\boldsymbol{\tau}^{c}+\boldsymbol{\tau}^{h}
$$

where $\boldsymbol{x} \in \mathbb{R}^{3}$ is the configuration vector, $M(\boldsymbol{x}) \in \mathbb{R}^{3 \times 3}$ the positive-definite and symmetric inertia matrix, $C(\boldsymbol{x}, \dot{\boldsymbol{x}}) \in \mathbb{R}^{3 \times 3}$ represents Coriolis and centrifugal terms, and $\left(\boldsymbol{\tau}^{c}, \boldsymbol{\tau}^{h}\right) \in \mathbb{R}^{3} \times \mathbb{R}^{3}$, are the control/human forces acting on the device, respectively. We assume, as usually done, that gravity is locally compensated.

The UAVs considered in this work belong to the quadrotor family: these represent a popular choice for a large number of research groups mainly because of their structural simplicity and mechanical robustness. A quadrotor can be conveniently modeled as a $3 \mathrm{D}$ rigid body with 3 torques and one force (the thrust) serving as control inputs, with the thrust being always oriented vertically in the body frame. Therefore the quadrotor is an underactuated system since only 4 inputs are available for controlling its 6 DOFs pose. Nevertheless the center of mass of the $i$-th quadrotor $\boldsymbol{p}_{i} \in \mathbb{R}^{3}$ and its yaw angle $\psi_{i} \in \mathbb{S}^{1}$ can be shown to be flat outputs [12, i.e., algebraically defining (with their derivatives) the state and the control inputs of the quadrotor [13. This makes it possible for quadrotor to track any smooth trajectory in the flat output space by using a suitable flight controller, see, e.g., 1214 . Owing to this property, every quadrotor of the group will be treated as a yaw-orientable $3 \mathrm{D}$ point in space $\left(\boldsymbol{p}_{i}, \psi_{i}\right)$.

In order to let the human interact with the quadrotors while ensuring a safe an cohesive navigation of the UAV group, we assume that $\boldsymbol{p}_{i}$ can be driven at the kinematic level by the sum of 3 velocity components, $\boldsymbol{u}_{i}^{h}, \boldsymbol{u}_{i}^{g}, \boldsymbol{u}_{i}^{e}$. These represent the human, group, and environmental control components of the total velocity command, respectively, thus resulting in:

$$
\dot{\boldsymbol{p}}_{i}=\boldsymbol{u}_{i}^{h}+\boldsymbol{u}_{i}^{g}+\boldsymbol{u}_{i}^{e}
$$

The human control is set as $\boldsymbol{u}_{i}^{h}=\lambda_{h} \boldsymbol{x}$ if $i \in M$ and $\boldsymbol{u}_{i}^{h}=\mathbf{0}$ if $i \notin M$, where $\lambda_{h} \in \mathbb{R}^{+}$is a scaling factor and $M$ is the set of UAVs in communication with the human operator. The group control term $\boldsymbol{u}_{i}^{g}$ is chosen in order to enforce the desired UAV formation as much as possible, e.g, by keeping desired interdistances or relative bearings, and $\boldsymbol{u}_{i}^{e}$ has the role of ensuring obstacle avoidance, e.g., by resorting to artificial potential approaches. The term $\boldsymbol{u}_{i}^{e}$ can also account for other environmental-related features, such as avoiding dangerous zones or being attracted by promising areas.

Every quadrotor may carry a camera onboard whose optical axis is parallel to the quadrotor frame. In these cases, we assume the yaw angle $\psi_{i}$ to be autonomously regulated so as to let the camera optical axis match with the horizontal direction of motion, e.g., by using the proportional controller:

$$
\dot{\psi}_{i}=K_{\psi}\left(\operatorname{atan} 2\left(\dot{\boldsymbol{p}}_{i y}, \dot{\boldsymbol{p}}_{i x}\right)-\psi_{i}\right)
$$

where $K_{\psi}$ is a positive gain. This allows to provide the operator with a forward view of the remote environment in case of quasi-horizontal motion. 


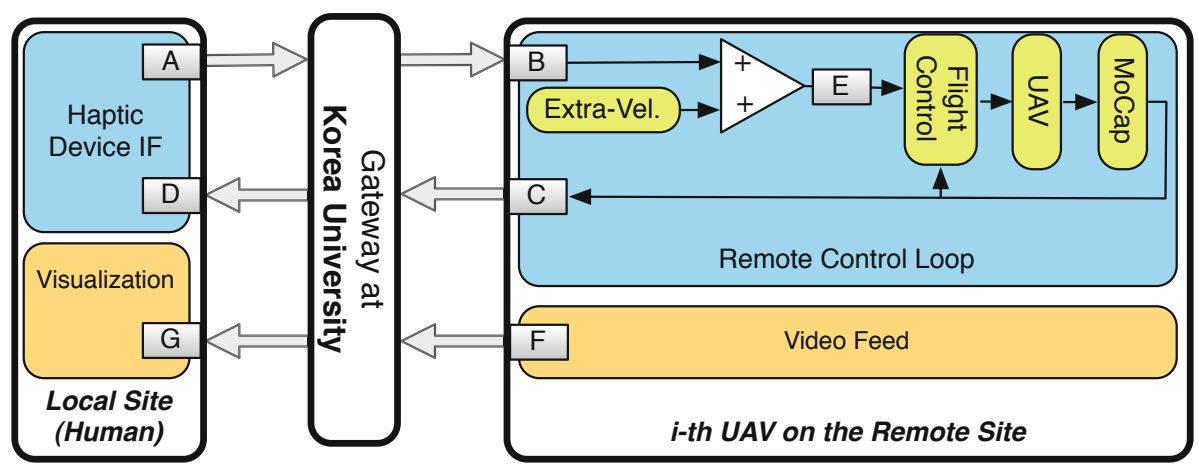

Fig. 1. Signals exchanged in the intercontinental teleoperation system. The relevant signals are labelled as follows: (A) human-commanded velocity at the local site; (B) delayed velocity input received by the $i$-th UAV at remote site; (C) state of the $i$-th UAV determined by motion-capture system; (D) delayed states of all UAVs utilized in the calculation of the appropriate haptic force; (E) actual commanded UAV velocity calculated from human-commanded velocity and artificial potential (obstacle avoidance and formation control $) ;(\mathrm{F} / \mathrm{G})$ videos transmitted/received from the remote to the local site.

In order to render to the human operator a haptic perception of the remote environment, we set the control torque as

$$
\boldsymbol{\tau}^{c}=-B \dot{\boldsymbol{x}}+K\left(\frac{1}{|M|} \sum_{i \in M} \dot{\boldsymbol{q}}_{i}-\lambda_{h} \boldsymbol{x}\right)
$$

where $B, K \in \mathbb{R}^{3 \times 3}$ are a positive semidefinite damping matrix and a positive gain matrix, respectively, and $\dot{\boldsymbol{q}}_{i}$ is the velocity of the real $i$-th quadrotor UAV. Apart from the stabilizing role of the term $B \dot{\boldsymbol{x}}$, the behavior of $\boldsymbol{\tau}^{c}$ is meant to represent the mismatch between the commanded velocity and the average velocity of the UAVs in $M$. This way, any external disturbance (e.g., wind gusts) can be perceived by the operator, as well as the presence of any obstacle obstructing the commanded direction of motion.

Several techniques have been proposed in the literature in order to ensure a stable behavior in presence of delays, packet losses, or other non-idealities in the communication channel between the master and slave sides of a bilateral teleoperation channel. In our transcontinental experiments, we made use of a conservative yet simple and effective method, namely, an appropriate tuning of the damping term $B$ in order to dissipate any energy excess. The application of more sophisticated techniques such as [1516] could provide less average perceptual degradation and their use is left as a future extension of this work. 


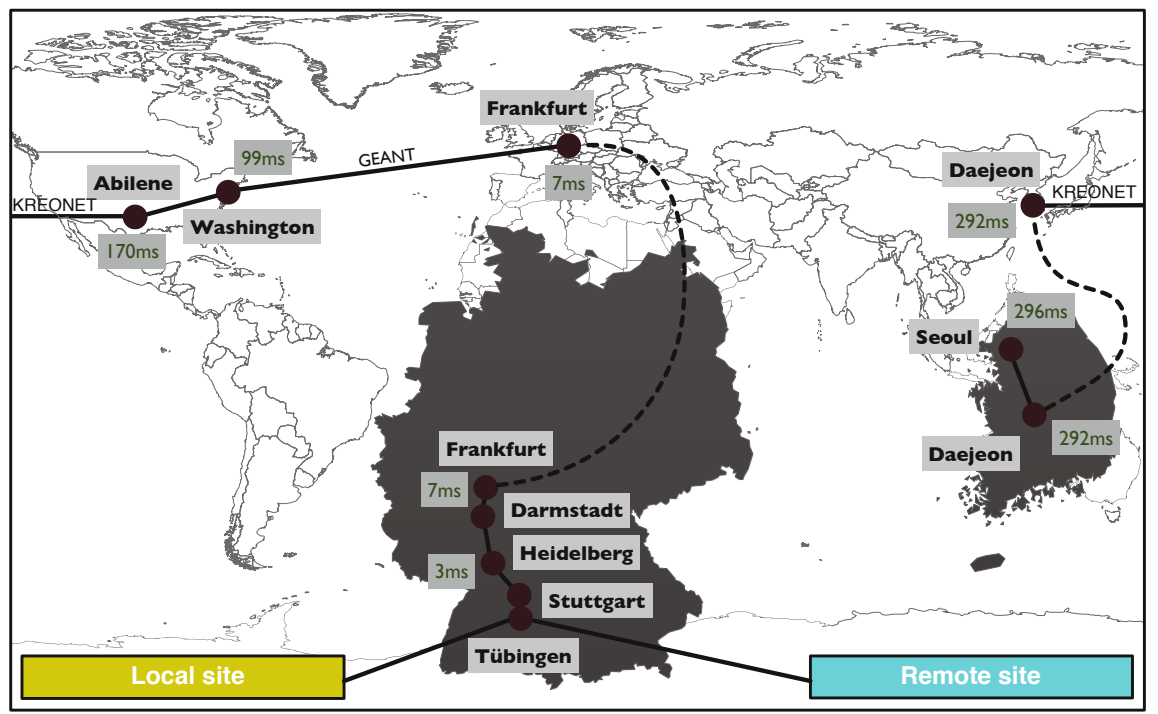

Fig. 2. Representation of the default packet routing and average delays between the MPI of Biological Cybernetics, Tübingen, and Korea University, Seoul. Some local hops were omitted to increase clarity.

\section{Experimental Setup}

Our experimental setup is made by two environments, namely, the remote (UAV), and the local (human operator) sites, see Fig. 1. These two environments are both located in a building at the Max Planck Institute for Biological Cybernetics, in Tübingen (MPI) but are connected exclusively by means of a real intercontinental Internet channel which passes through a machine located at the Korea University, in Seoul (KU). Tracing packets between both endpoints, revealed a route over the North American continent containing 25 hops resulting in a raw IMCP travel time of about $295 \mathrm{~ms}$, as depicted in Fig. 2] We established a site-to-site OpenVPN1 connection (dev: tap, proto: udp) between a subnet at the MPI and the machine at KU in order to allow for secure communication, without sacrificing performance (raw single-trip time over the VPN: $305 \mathrm{~ms}$ ).

On the remote site we use 2 quadrotors 2 as UAVs, for which we developed a custom-made software and hardware setup, see Fig. 3, right. A motion capture system 3 with six cameras monitors the remote-site flight area of $3 \times 4 \times 3 \mathrm{~m}$ and provides position and orientation of each UAV with a frequency of $120 \mathrm{~Hz}$ with about $10 \mathrm{~ms}$ of delay. Almost all the computation on the remote site is done on a six-core $\operatorname{Intel}(\mathrm{R}) \mathrm{Xeon}(\mathrm{R}) \mathrm{PC}$ and communication to the UAVs is provided by a

\footnotetext{
$1 \longdiv { \text { openvpn.net } }$

2 mikrokopter.de

3 vicon. com
} 


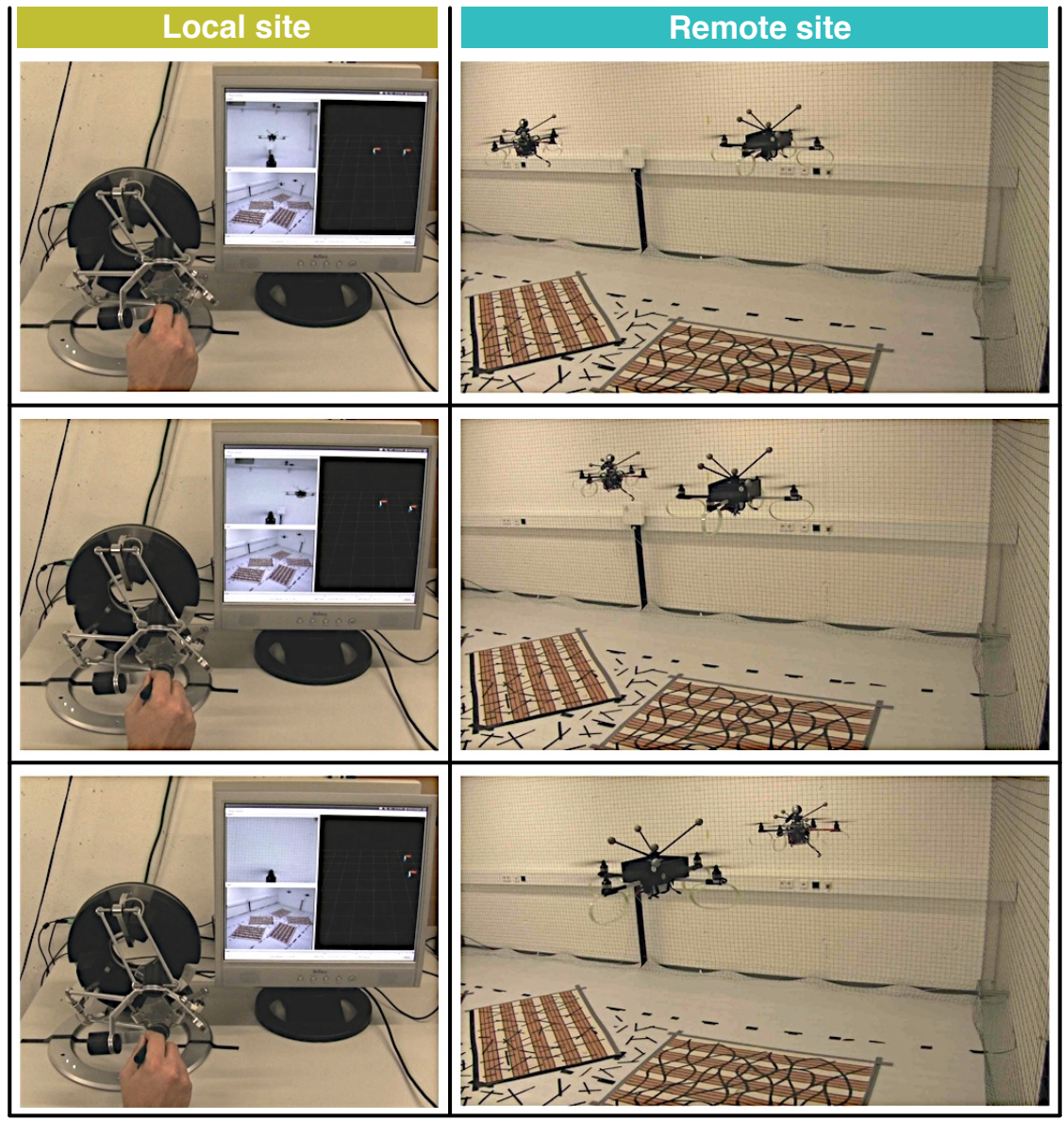

Fig. 3. A representative experiment of intercontinental bilateral teleoperation with 2 quadrotor UAVs. Left column: the local (human operator) site. Right column: the remote (UAV) environment. Each row represents a different time instant. The human operator is provided with a haptic interface in order to control the overall motion of the UAVs and 3 video streams: an onboard view, a global view, and a 3D representation of the UAV states. The two sites are connected through an intercontinental channel implementing a full Germany-South Korea roundtrip.

wireless serial connection XBee-PRO 802.15.4. A separate Intel(R) Pentium(R) 4 gateway $\mathrm{PC}$ with $1 \mathrm{~Gb}$ optical fiber connection to the Internet and $1 \mathrm{~Gb}$ Ethernet to the local network provides routing, firewall and VPN capabilities. One of the UAVs is also equipped with a Q7 board holding an Intel Atom 1.6Ghz CPU to wirelessly transmit image data from an onboard camera, or to run the flight controller without the explicit need of a base station. In addition to the onboard camera we capture the remote site arena also using a wall-mounted fixed camera. Both cameras send a low-resolution MJPEG-encoded video stream (160x120) at 
$10 \mathrm{fps}$ to the operator site. A standard USB gamepad is used as console in order to control the experimental flow (e.g., liftoff, operate, land, emergency...).

On the local site we use an Omegat as haptic device, which provides 3 actuated DOFs. For our remote setup, an Asus WL-500gP with customized TomatoUSB5 enables us to establish a site-to-site VPN between the router and our local network from any valid IPv4 Internet connection. We are also able to initiate a road-worrier connection directly from a client computer.

We control the 2 quadrotors over the Internet by implementing the approach described in Sec. 2 Both the UAVs keep a formation by following an independent reference trajectory that is a result of the tele-operators input velocity and an artificial potential (providing connectivity preservation and obstacle avoidance), as expressed in (2). UAV State information (position, orientation velocity, and angular velocity) is transmitted over the Internet to the haptic device in order to compute the force feedback (4) which is informative of the remote UAV performance. In addition, we provide the remote operator with a $3 \mathrm{D}$ visualization of the quadrotors body-frames in the world frame and two video streams, transmitting an overall view of the flying area and a first-person representation along the axis of one UAV, see Fig. 3, left. By using (3) the optical axis of the camera is able to naturally follow the direction of the user-commanded motion.

We developed a custom framework based on the Robot Operating System 6 (ROS) that simplifies the implementation of multi-robot control algorithms by abstracting all necessary functionality into distinct user-loadable modules. This results in versatile applications, like the intercontinental bilateral control of multiple UAVs. As in ROS, algorithms, drivers and sensors are split into distinct programs, that provide or receive information from and to the rest of the infrastructure.

The software setup is divided into local processes, passing from Germany through the gateway in South Korea and back, and remote processes that are solely executed in the remote subnet, which are schematized in Fig. 1 from the perspective of the $i$-th $\mathrm{UAV}$.

Locally, the ROS tool rviz provides the video streams and presents a 3D Interface to the human operator. Moreover, the haptic device control process, running at $3 \mathrm{kHz}$, sends the desired velocity commands at a rate of $100 \mathrm{~Hz}$ and receives state information at about $80 \mathrm{~Hz}$ from both UAVs to calculate an appropriate haptic feedback (these messages are also used to refresh the 3D Interface).

At the same time, on the remote site, one instance of a custom closed-loop controller for each UAV implements the formation control algorithm and publishes the state information to the other processes at the local site. We interface our quadrotors with a process abstracting the serial communication into the ROS infrastructure. Dedicated applications map the console inputs as well as the video streams into ROS.

\footnotetext{
${ }^{4}$ forcedimension.com

5 tomatousb.org

6 ros.org
} 


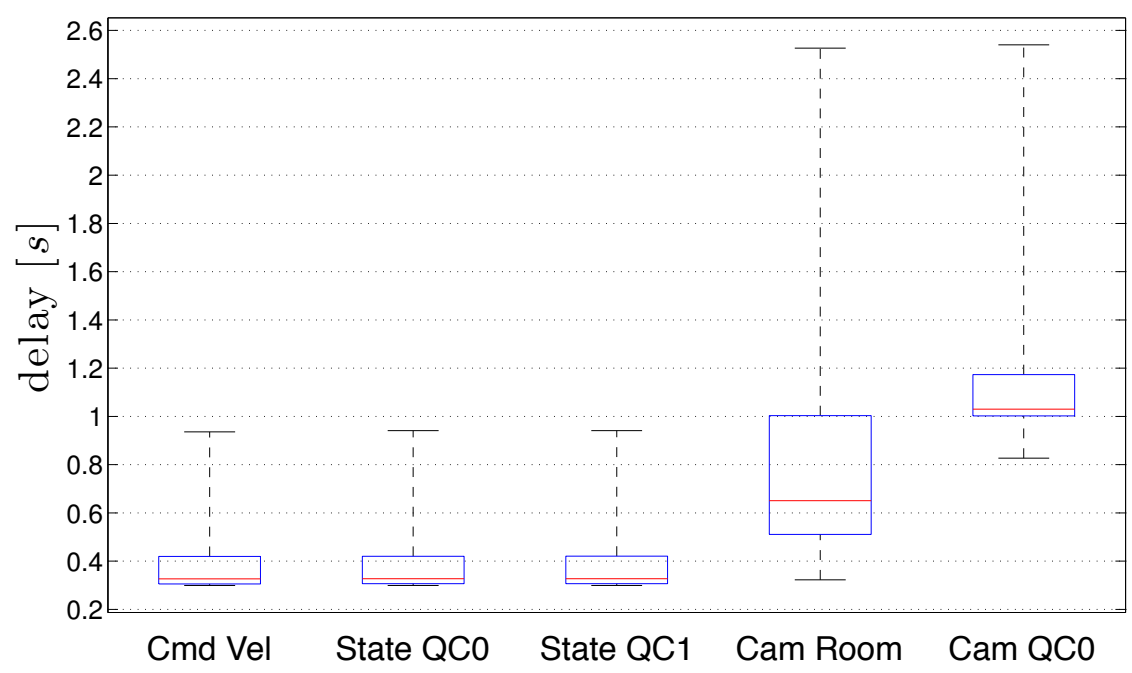

Fig. 4. Boxplots describing the (round-trip) delay times for all messages passing over the gateway at Korea University. Middle bars indicates the median values, while boxes and and whiskers denote the two median quartiles and the maximum-minimum values respectively. Bilateral control-loop messages, i.e., the commanded velocity (Cmd Vel) and the states of both UAVs (State QC0 and State QC1) are transmitted with an average delay of about $350 \mathrm{~ms}$. Video streams are transmitted with the higher average lags of $650 \mathrm{~ms}$ and $1 \mathrm{~s}$ for the wall-mounted camera (Cam Room) and onboard camera (Cam QC0) respectively. The delay of the onboard camera stream is larger compared to the room video because of the additional wireless link.

\section{Results and Discussion}

We describe now the results of a representative experiment whose main phases are illustrated in the sequence of Fig. 3. At the beginning of the experiment the UAVs are steered by the human operator toward a wall, the first UAV then immediately stops, thus generating a haptic feedback to the operator. By continuing to apply the desired velocity against that force, the UAVs rearrange and the centroid of the formation is able to move closer to the wall. This event gets fed back to the human by means of a releasing of the set force. A video summary of our intercontinental teleoperation experiment is located at http://antoniofranchi.com/links/2012-IAS12-InterContTele0p.html

As explained in the previous sections, the human operator transmits velocity commands to the remote site by setting the appropriate position on the haptic device. The commands are received by the flight controller of each UAV with varying delay of around $350 \mathrm{~ms}$ (see Fig. 4) and added as in (2) in order to calculate the actual UAV velocity reference (see Fig. 1). Fig. 可(a) direclty compares sent and received commands, where the delay is clearly visible. The flight controller then computes the appropriate UAV propeller speeds in order to track that velocity reference with good performances, as shown in the comparison of Fig. 国(b). 


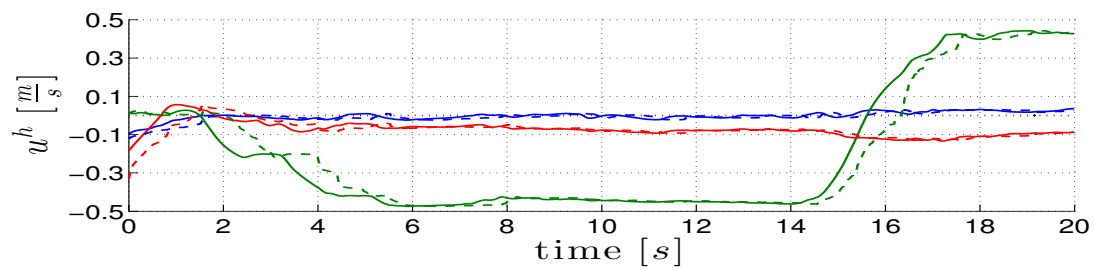

(a)

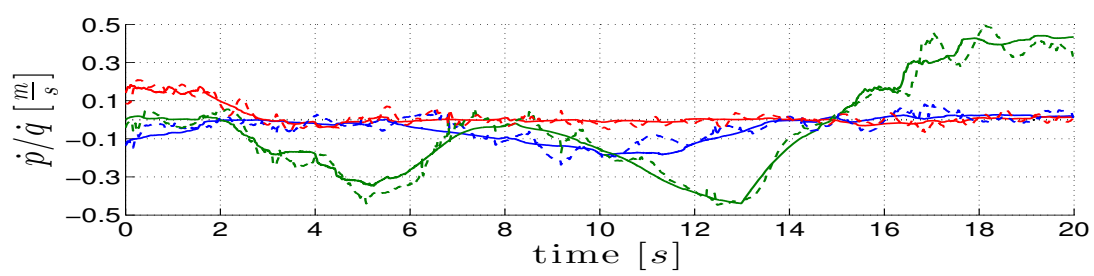

(b)

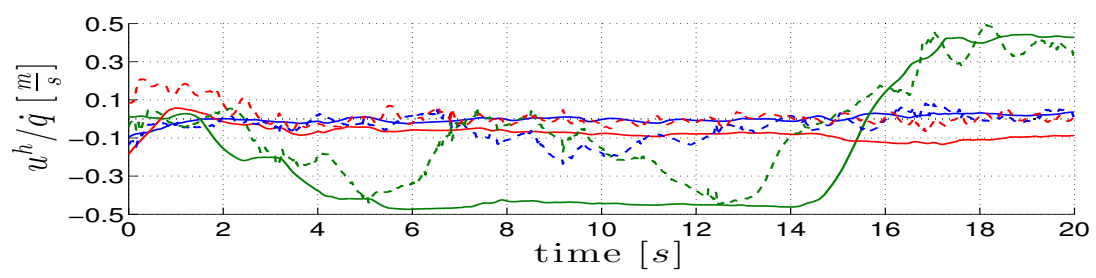

(c)

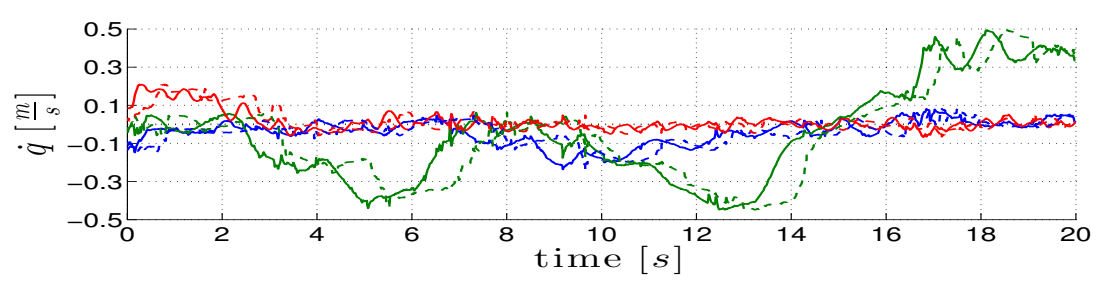

(d)

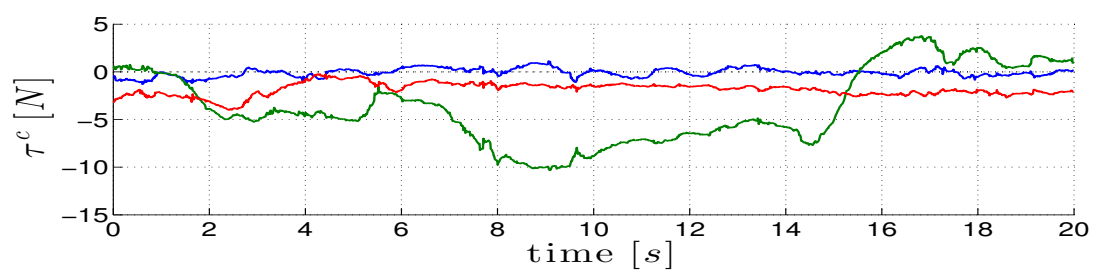

(e)

Fig. 5. A representative experiment of intercontinental bilateral teleoperation with 2 quadrotor UAVs. (a) velocity commanded by the human operator $\boldsymbol{u}^{h}$, sent (solid) and received (dashed) with delay; (b) desired velocity velocity $\dot{\boldsymbol{p}}$ (solid) versus actual UAV velocity $\dot{\boldsymbol{q}}$ (dashed); (c) commanded velocity $\boldsymbol{u}^{h}$ (solid) versus actual UAV velocity $\dot{\boldsymbol{q}}$ (dashed); (d) actual UAV velocity $\dot{\boldsymbol{q}}$ : sent (solid) and received (dashed) with delay: (e) force feedback $\tau^{c}$. 
On the local site the haptic device is constantly receiving state information of both UAVs at a rate of $80 \mathrm{~Hz}$ with still a time-varying delay of $350 \mathrm{~ms}$ on average (see Fig. 4 for a statistical analysis of the delay and Fig. 馬(d) for a direct comparison between sent and received state signals for one of the two UAVs.). The desired velocity passed to the flight controller $\dot{\boldsymbol{p}}$ is compared to the one commanded by the operator $\boldsymbol{u}^{h}$ in Fig. 馬(c) where it is possible to appreciate the mismatch caused by the influence of the additional contributions given by the formation control and obstacle avoidance terms in (2). That error is used to implement a proportional force feedback, depicted in Fig. 5 (e) which therefore has a dual functionality: first it informs the user about the UAV inertia and delay of the communication system, and second it indicates the influence of environmental factors on the UAV dynamics, e.g., presence of obstacles, turbulence, etc.

The transmissions of both video streams suffer of higher latencies compared to the control/haptic-related messages, as clear in Fig. 4. The video stream from the wall-mounted camera has an average delay of $650 \mathrm{~ms}$ while the video from the onboard camera experiences an average delay of about $1 \mathrm{~s}$, because of the additional WiFi link. Both video streams have also a much wider variability in the delay when compared with the other signals, with peaks reaching $2.5 \mathrm{~s}$.

In conclusion, the experimental results demonstrate the advantages provided by the use of a haptic feedback beside the visual one in the following way: (1) velocity commands and state messages are transmitted faster and more reliably compared to a video stream and (2) the view angle of $3 \mathrm{D}$ environments and camera setups does not always give an accurate representation of distances, which can instead be provided more promptly by the use of suitable haptic cues.

The interested reader can find additional videos concerning the teleoperation of multiple UAVs in different scenarios at http://www.youtube.com/user/ MPIRobotics/videos.

\section{Conclusions and Future Work}

In this notes, we presented experiments on the intercontinental bilateral control of a group of UAVs, by relaying packets between Germany and South Korea, forwarding velocity commands to the remote site and sending state information for haptic feedback and visualization purposes back to the local operator. Additionally, we transmitted two video streams to give the operator a complete representation of the remote site. With a average delay of about $350 \mathrm{~ms}$ we were able control the UAVs in a stable and reliable manner.

In the future we would like to apply our implementation of intercontinental teleoperation to other teleoperation algorithms and evaluate their performance and robustness in real-world conditions. Our scenario implements a round-trip teleoperation between Europe and Asia, effectively doubling the delay compared to a direct communication channel. We plan to demonstrate our setup with a more standard single-trip delay in a live demo at IAS-12 in South Korea. 
Acknowledgments. The authors like to thank Johannes Lächele for creating the simulator and sharing his technical expertise during the creation of the software setup, and the people from Korea University for providing us the access to the relay machine on their campus.

This research was partly supported by WCU (World Class University) program funded by the Ministry of Education, Science and Technology through the National Research Foundation of Korea (R31-10008).

\section{References}

1. Valavanis, K.P.: Advances in Unmanned Aerial Vehicles: State of the Art and the Road to Autonomy, vol. 33 (2007)

2. Murray, R.M.: Recent research in cooperative control of multi-vehicle systems 129(5), 571-583 (2007)

3. Michael, N., Fink, J., Kumar, V.: Cooperative manipulation and transportation with aerial robots, Seattle, WA (June 2009)

4. Michael, N., Mellinger, D., Lindsey, Q., Kumar, V.: The GRASP multiple microUAV testbed $17(3), 56-65$ (2010)

5. Hokayem, P.F., Spong, M.W.: Bilateral teleoperation: An historical survey 42(12), 2035-2057 (2006)

6. Rodríguez-Seda, E.J., Troy, J.J., Erignac, C.A., Murray, P., Stipanović, D.M., Spong, M.W.: Bilateral teleoperation of multiple mobile agents: Coordinated motion and collision avoidance 18(4), 984-992 (2010)

7. Lee, D., Franchi, A., Robuffo Giordano, P., Son, H.I., Bülthoff, H.H.: Haptic teleoperation of multiple unmanned aerial vehicles over the internet, Shanghai, China, pp. 1341-1347 (May 2011)

8. Franchi, A., Secchi, C., Son, H.I., Bülthoff, H.H., Robuffo Giordano, P.: Bilateral teleoperation of groups of mobile robots with time-varying topology (accepted to 2012)

9. Marescaux, J., Leroy, J., Gagner, M., Rubino, F., Mutter, D., Vix, M., Butner, S.E., Smith, M.K.: Transatlantic robot-assisted telesurgery 413, 379-380 (2001)

10. King, H.H., Hannaford, B., Kwok, K.-W., Yang, G.-Z., Griffiths, P., Okamura, A., Farkhatdinov, I., Ryu, J.-H., Sankaranarayanan, G., Arikatla, V., Tadano, K., Kawashima, K., Peer, A., Schauss, T., Buss, M., Miller, L., Glozman, D., Rosen, J., Low, T.: Plugfest 2009: Global interoperability in telerobotics and telemedicine, Anchorage, AK, pp. 1050-4729 (May 2010)

11. Bolopion, A., Stolle, C., Tunnell, R., Haliyo, S., Régnier, S., Fatikow, S.: Remote microscale teleoperation through virtual reality and haptic feedback, San Francisco, CA, pp. 894-900 (September 2011)

12. Mistler, V., Benallegue, A., M'Sirdi, N.K.: Exact linearization and noninteracting control of a 4 rotors helicopter via dynamic feedback, Bordeaux, Paris, France, pp. 586-593 (September 2001)

13. Fliess, M., Lévine, J., Martin, P., Rouchon, P.: Flatness and defect of nonlinear systems: Introductory theory and examples 61(6), 1327-1361 (1995) 
14. Lee, T., Leokyand, M., McClamroch, N.H.: Geometric tracking control of a quadrotor UAV on SE(3), Atlanta, GA, pp. 5420-5425 (December 2010)

15. Lee, D.J., Huang, K.: Passive-set-position-modulation framework for interactive robotic systems 26(2), 354-369 (2010)

16. Franken, M., Stramigioli, S., Misra, S., Secchi, C., Macchelli, A.: Bilateral telemanipulation with time delays: A two-layer approach combining passivity and transparency 27(4), 741-756 (2011) 